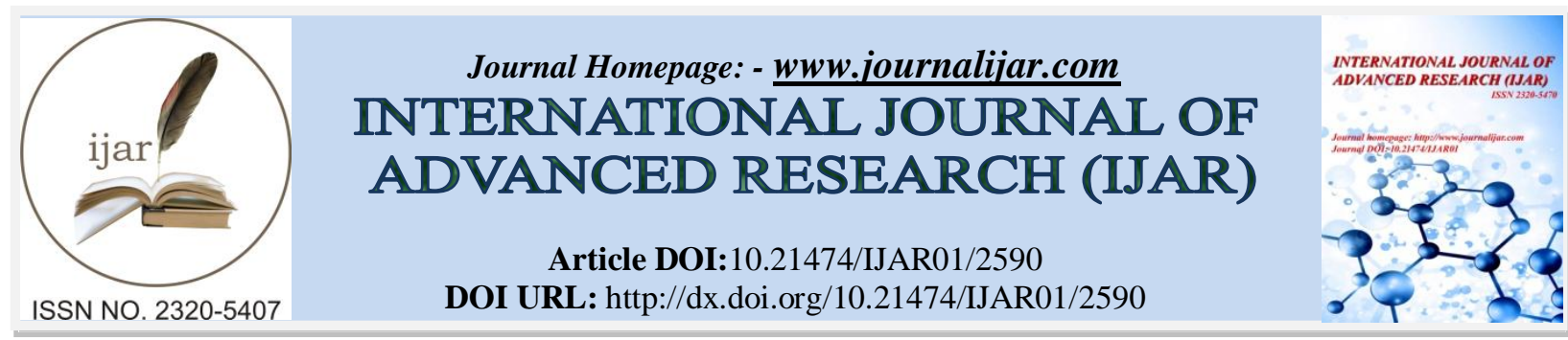

RESEARCH ARTICLE

\title{
STAT4 (RS7582694) GENE POLYMORPHISM IN A GROUP OF EGYPTIAN FEMALE SYSTEMIC LUPUS ERYTHEMATOSUS PATIENTS.
}

\author{
Mona El-Toukhy ${ }^{1}$, Ibrahim Rageh ${ }^{1}$, Eman Abdel-Gwad ${ }^{1}$, Amal Fathy ${ }^{2}$, Ola El-Shimi ${ }^{1 *}$ and Marian Roshdy ${ }^{3}$. \\ 1. Clinical \& Chemical Pathology department, Faculty of Medicine, Benha University, Benha, Egypt. \\ 2. Rheumatology department, Faculty of Medicine, Benha University, Benha, Egypt. \\ 3. Quwisna Central Hospital, Ministry of Health,Quwisna, Egypt.
}

\section{Manuscript Info}

Manuscript History

Received: 27 October 2016

Final Accepted: 25 November 2016

Published: December 2016

Key words:-

Egyptians, rs7582694, SLE, STAT4.

\section{Abstract}

Systemic lupus erythematosus (SLE) is a chronic complex autoimmune disease. It results from interaction of genetic, environmental and hormonal factors. Genome Wide Association (GWA) studies have proved the association of multiple STAT4 gene polymorphisms with SLE in different populations. Subjects \& methods: The study enrolled 50 Egyptian females, classified into 2 groups: 30 SLE patients and 20 apparently healthy individuals. STAT4 gene C>G (rs7582694) polymorphism was analyzed by PCRRFLP (Polymerase chain reaction - Restriction fragment length polymorphism) technique. Results: The frequency of minor allele $\mathrm{C}$ of the STAT4 (rs7582694) polymorphism was higher in SLE patients than control group but with no statistically significant difference between the two studied groups $(\mathrm{P}=0.518)$. There was neither association between genotypes and SLE nor SLEDAI activity grades. Conclusion:STAT4 gene C>G (rs7582694) polymorphism is not associated with the predisposition to SLE or disease activity in the studied group of Egyptian SLE patients.

Copy Right, IJAR, 2016,All rights reserved.

\section{Introduction:-}

Systemic lupus erythematosus (SLE, or lupus) is a complex systemic autoimmune disease with multi-organ involvement. It occurs in genetically-predisposed individuals who have exposed to certain environmental or random stimuli (Deng and Tsao, 2010).SLE has a sole pattern of incidence, being more common in females than males, with a ratio ranges from 4 to 12:1. It could occur in all ages, but the peak age of onset is during childbearing years (Hu et al., 2016).SLE is characterized by the production of pathogenic autoantibodies directed against nucleic acids and their binding proteins, which resulting from a global loss of self-tolerance (Choi et al., 2012). This loss of selftolerance with subsequent immune dysregulation is a result of interaction of predisposing genetic factors with a set of environmental and hormonal triggers. The studies associate over 30 genetic loci with the disease pathogenesis (Delgado-Vega et al., 2010).

STAT4 (signal transducers and activators of transcription-4) is a transcription factor transducing IL-12, IL-23, and type 1 interferon-mediated signals for Th1 and Th17 differentiation, monocyte activation, and interferon-gamma production (Glas et al., 2010).

Corresponding Author:-Ola El-Shimi.

Address:-Clinical \& Chemical Pathology department, Faculty of Medicine, Benha University, Benha, 
STAT4gene polymorphismshave been found to be associated with SLE in multiple Genome Wide Association Studies (GWAS)in populations of European or Asian ancestry (Harley et al., 2008; Graham et al., 2008; Han et al., 2009; Yang et al., 2010).However; limited data is available about its association with SLE patients from different origins. Thus, we investigated the association of STAT4 gene C>G (rs7582694) polymorphism with SLE in Egyptians.

\section{Materials and Methods:- Study population:-}

This study was reviewed and approved by the local ethics committee of Faculty of Medicine, Benha University, Egypt. We recruited 30 unrelated adult female Egyptian patients with SLE from the Rheumatology department at

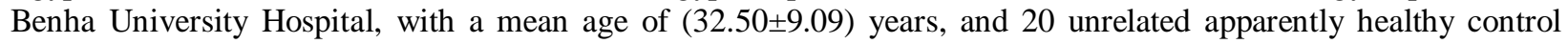
subjects coming from the same geographical area with a mean age of (30.60土8.7) years, with no past or family history of SLE or any other disorder. An informed written consent was obtained from each participant. All enrolled patients fulfill four or more of the eleven criteria designated by the American College of Rheumatology (ACR) for the diagnosis of SLE (Tan et al., 1982).

All individuals were subjected to full history taking with special emphasis on the history of autoimmune diseases, systemic physical examination and detailed rheumatologic examination to examine the presence of the ACR criteria of SLE and to evaluate the disease activity by SLE Disease Activity Index(SLEDAI) score, laboratory investigations includingCBC, ESR, serum ANA, serum anti-dsDNA, serum C3, serum C4 and urinary albumin/creatinineratio.

\section{Sample collection and DNA isolation:-}

Peripheral venous blood samples were drawn from SLE patients and control group in $5 \mathrm{~mL}$ sterile syringes using aseptic venipuncture technique and transferred immediately to EDTA coated vacutainers to prevent coagulation. Genomic DNA was extracted using a GeneJET Whole Blood Genomic DNA Purification Mini Kit supplied by Thermo Scientific, (Cat. No. K0781, Germany) according to manufacturer protocol.

\section{Genotyping:-}

The STAT4 gene $C>G$ (rs7582694) polymorphism was genotyped by polymerase chain reaction using the primer pairFP: 5'-ATCCAACTCTTCTCAGCCCTT-3', RP: 5'-TCATAATCAGGAGAGAGGAGT-3', and conditions of PCR cycles were set as follows: an initial denaturation temperature of $95^{\circ} \mathrm{C}$ for $1 \mathrm{~min}$, followed by 35 cycles of $95^{\circ} \mathrm{C}$ for $15 \mathrm{sec}, 55^{\circ} \mathrm{C}$ for $15 \mathrm{sec}$ and $72^{\circ} \mathrm{C}$ for $10 \mathrm{sec}$ followed by final extension at $72^{\circ} \mathrm{C}$ for $10 \mathrm{~min}$. Amplified fragment of $338 \mathrm{bp}$ was digested with Taalfast digest (5 ...ACN $\downarrow$ GT ...3 and $3^{\circ} \ldots$ TG $\left.\uparrow N C A \ldots 5^{\prime}\right)$ at $65^{\circ} \mathrm{C}$ for 5 min, supplied byThermoScientific, (Cat. No. FD1364, Germany). The STAT4 (rs7582694)C allele was cleaved into 258 and 80 bp fragments, whereas the STAT4 (rs7582694)G allele remained uncut at 338 bp (Figure 1).

\section{Statistical analysis:-}

The clinical characteristics (i.e., age and gender parameters) of the patient and control groups were compared using Student's $t$ test. The Chi square $\left(\mathrm{X}^{2}\right)$ and Fisher's exact tests were used to compare genotype and allele frequencies between the control and patient groups.Deviations from Hardy-Weinberg equilibrium expectations were determined using the chi-squared test. GG genotype and G allele were considered as references.Logistic and ordinal regression analysis were done for prediction of risk factors. Statistical significance was established at $\mathrm{P}<0.05$. Odds ratios (OR) for the strength of association and risk of SLE and their 95\% confidence intervals (CI)were calculated and the statistical tests were carried out using Microsoft Excel 2013 and Statistical Package for Social Science version 20.0 software (SPSS Inc., Chicago, IL,USA).

\section{Results:-}

Our studied sample of individuals was selected randomly from population in Kaluobia Governorate in Lower Delta, Egypt. By applying the Hardy Weinberg equation (HWE), the STAT4 (rs7582694) genotypes in both case and controlsubjects were independent $(\mathrm{P}>0.05)$. Genotypic and allelic distribution and association analysis of STAT4 (rs7582694) among SLE patients and control groups are shown in Table (1). The frequency of the minor allele C was observed in $13.3 \%$ of SLE patients and in $7.5 \%$ of apparently healthy controls but without significant association ( $\mathrm{P}=0.518$ ). While, the genotypes $\mathrm{GG}$ and $\mathrm{GC}$ were observed in $73.3 \%$ and $26.7 \%$ respectively in SLE patients and $85 \%$ and $15 \%$ respectively in apparently healthy individuals without significant association $(\mathrm{P}=$ 0.489).When we analyzed the genotypic distribution among the different degrees of activity of SLE, STAT4 
(rs7582694) genotypes did not show significant differences between various SLEDAI activity grades (P=0.199) Table (2). When we compared between STAT4 (rs7582694) genotypes regarding ACR criteria in studied SLE group, no statistically significant differences could be observed (data not shown). When we applied age, disease duration, anti-dsDNA, albumin/creatinine ratio, C3, C4 concentrations and STAT4 (rs7582694) genotypes as covariates; the univariate analysis revealed that anti-dsDNA and albumin/creatinine ratio were significantly associated with the risk of increased SLEDAI score, while higher C3, C4 concentrations were significantly associated with lower SLEDAI score. However; in multivariate analysis, taking those variables that showed significant associations in univariate analysis, only anti-dsDNA and albumin/creatinin ratio were significantly associated with the risk of increased SLEDAI score Table (3).

Table 1:- Distribution of STAT4 (rs7582694) (alleles and genotypes) in SLE patients and control subjects:

\begin{tabular}{|c|c|c|c|c|c|c|c|c|}
\hline & & \multicolumn{2}{|c|}{$\begin{array}{l}\text { Control } \\
(n=20)\end{array}$} & \multicolumn{2}{|c|}{$\begin{array}{c}\text { SLE } \\
(n=30)\end{array}$} & \multirow[t]{2}{*}{$\mathrm{P}$} & \multirow[t]{2}{*}{ OR } & \multirow[t]{2}{*}{$95 \% \mathrm{CI}$} \\
\hline & & $\mathrm{N}$ & $\%$ & $\mathrm{~N}$ & $\%$ & & & \\
\hline \multirow[t]{2}{*}{ Genotypes } & GG & 17 & 85 & 22 & 73.3 & \multirow[t]{2}{*}{0.489} & \multirow[t]{2}{*}{2.061} & \multirow[t]{2}{*}{$(0.474-8.963)$} \\
\hline & $\mathrm{GC}$ & 3 & 15 & 8 & 26.7 & & & \\
\hline \multirow[t]{2}{*}{ Alleles } & $\mathrm{G}$ & 37 & 92.5 & 52 & 86.7 & \multirow[t]{2}{*}{0.518} & \multirow[t]{2}{*}{1.897} & \multirow[t]{2}{*}{$(0.472-7.635)$} \\
\hline & $\mathrm{C}$ & 3 & 7.5 & 8 & 13.3 & & & \\
\hline
\end{tabular}

SLE: systemic lupus erythematosus, P: P-value, OR: odd ratio, CI: confidence interval

Table 2:- Comparison between STAT4 (rs7582694) genotypes regarding SLEDAI grades in studied SLE group.

\begin{tabular}{|c|c|c|c|c|c|c|}
\hline \multirow{2}{*}{\multicolumn{2}{|c|}{ SLEDAI }} & \multicolumn{5}{|c|}{ SLE } \\
\hline & & \multicolumn{2}{|c|}{$\begin{array}{c}\mathrm{GG} \\
(\mathrm{n}=22)\end{array}$} & \multicolumn{2}{|c|}{$\begin{array}{c}\mathrm{GC} \\
(\mathrm{n}=8)\end{array}$} & $\mathrm{P}$ \\
\hline \multicolumn{2}{|c|}{ Score; median (range) } & 7 & $0-32$ & 6.5 & $0-30$ & 0.585 \\
\hline \multirow[t]{3}{*}{ Grades; N (\%) } & Inactive & 6 & 27.3 & 2 & 25 & \multirow[t]{3}{*}{0.199} \\
\hline & Mild to moderate & 6 & 27.3 & 5 & 62.5 & \\
\hline & Severe & 10 & 45.5 & 1 & 12.5 & \\
\hline
\end{tabular}

SLEDAI: Systemic Lupus Erythematosus Disease Activity Index, SLE: systemic lupus erythematosus, P: P-value

Table 3:- Ordinal regression analysis for prediction of activity of SLE (assessed by SLEDAI score).

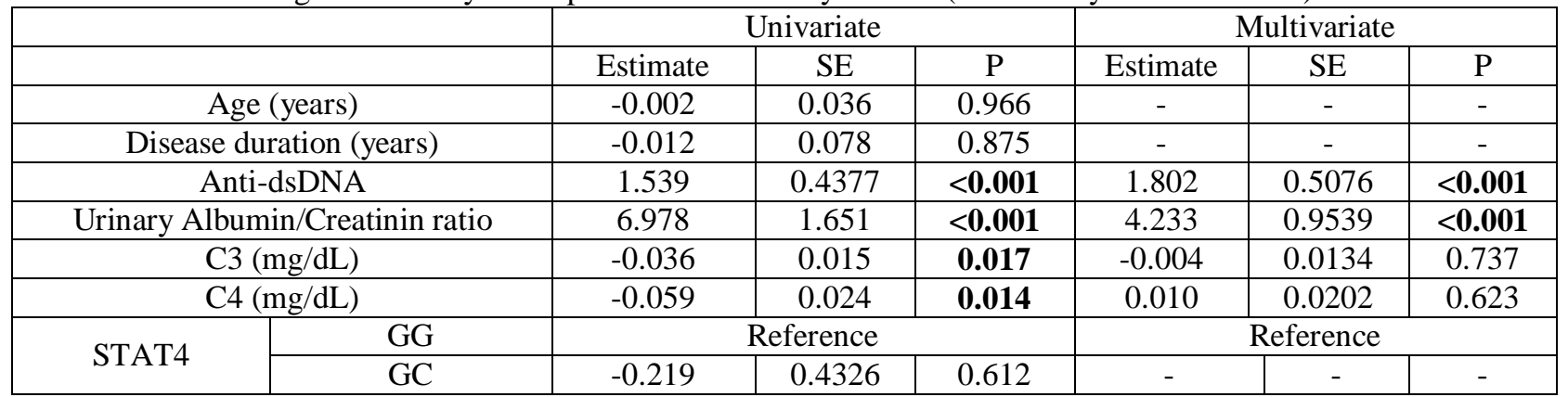

SE: standard error, P: P-value 


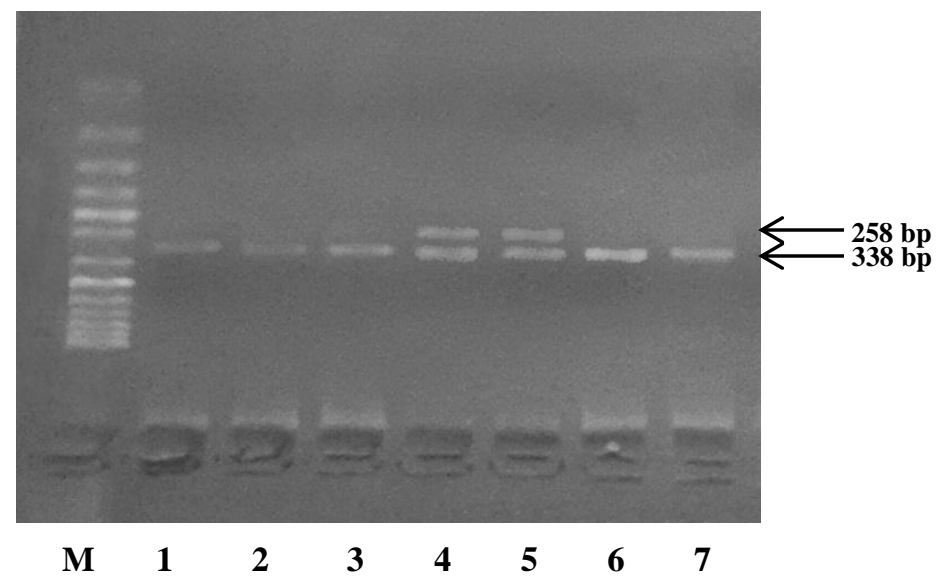

Fig 1:-Agarose gel electrophoresis pattern of rs7582694 polymorphism of STAT4 gene. Lane M: A 50 bp marker.

Lanes 1, 2 and 3: The PCR amplification product of target gene.

Lanes 4 and 5: The heterozygous GC genotype.

Lanes 6 and 7: The homozygous GG genotype.

\section{Discussion:-}

SLE is the prototypical chronic systemic autoimmune disease. It is characterized mainly by the production of autoantibodies against multiple cellular antigens including intracellular, nucleic acid and cell surface antigens (Alarcón-Riquelme et al., 2016). SLE is a multifactorial disease thus it has a complex pathogenesis, with several susceptibility genes, hormonal and environmental factors involved in its development and clinical manifestations (Ciccacci et al., 2014).Many genes were identified for their possible role in susceptibility to SLE.GWAS confirmed the genetic associations of more than 40 genes with SLE (Cui et al., 2013).Most of the described genes encode proteins that contribute in key pathogenic pathways, including Toll-like receptor and type I interferon signaling pathways, immune regulation pathways and clearance of immune complexes pathway (Deng and Tsao, 2010).STATproteins are major components in interferon (IFN)-dependent gene expression and are responsible for signal transduction of more than 50 cytokines, hormones, and growth factors which are a key regulators of cell survival, proliferation, and differentiation (Goropevšek et al., 2016). STAT4has been found to be associated with several autoimmune diseases, including rheumatoid arthritis (RA) and SLE (BeltránRamírez et al., 2016).Here, we studied the association of the STAT4 gene (rs7582694) polymorphism in a group of female Egyptian SLE patients and matched controls. We found that the minor allele $\mathrm{C}$ was higher in SLE patients than controls but without statistical significance $(\mathrm{P}=0.518)$. Regarding the genotype distribution among studied groups, both $\mathrm{GG}$ and GC genotypes were observed in $73.3 \%$ and $26.7 \%$ respectively in SLE patients and $85 \%$ and $15 \%$ respectively in healthy individuals but without significant association $(\mathrm{P}=0.489)$. When we compared different genotypes in patients with different disease activity indices according to SLEDAI score, we recognized that STAT4 (rs7582694) GG genotype was more in patients with severe grade of SLE (45.5\%). While, STAT4 (rs7582694) GC genotype was observed more in patients with mild to moderate grade of the disease $(62.5 \%)$.However this was not statistically significant $(\mathrm{P}=0.199)$.

Our results were in line with Luan et al. (2012); who observedno association between STAT4 (rs7582694) gene polymorphism and SLE or any clinical presentations in Chinese female population.STAT4 (rs7582694) gene polymorphismwas also confirmed in a Northern Han Chinese population not to be associated with SLE (Li et al., 2011).

On the contrary, Zhou and his colleague (2014); in the meta-analysis study that wasperformed to evaluate the relationship between STAT4gene polymorphisms and SLE / Lupus Nephritis (LN), they had found that among the studied SNPs the STAT4 (rs7582694) minor allele frequency was associated with the risk of SLE. Also, Piotrowski et al. (2012) confirmed an association of the STAT4 C (rs7582694) variant with the development of SLE and occurrence of some clinical manifestations of the diseasein Polish population.Moreover, a transmission disequilibrium test analysis proved a strongest signal of association for two linked STAT4 SNPs(rs7582694and rs10181656) in a Finnish SLE family cohort (Hellquist et al., 2010).Also, in the case-only meta-analysis, the STAT4 
SNP rs7582694 was found to be associated with severe renal insufficiency and to predispose to lupus nephritis (Bolin et al., 2013).Similarly,STAT4 rs7582694 polymorphism was strongly associated with SLE in Swedish patients and the risk allele of it was correlated to the production of anti-dsDNA antibodies (Sigurdsson et al., 2008).

The STAT4 (rs7582694) polymorphism have been proved to be associated with other autoimmune diseases such as primary Sjögren's Syndrome (pSS) in Sweden and Norway population (Nordmark et al., 2009) and in another Caucasian cohort (Gestermann et al., 2010). Whereas, The STAT4 (rs7582694) polymorphism was found to be not significantly associated with inflammatory bowel disease (IBD) (Zhu et al., 2013).

Other STAT4gene polymorphisms are proved to be associated with SLE as well. TheSTAT4rs7574865G/T polymorphism confers risk for RA andSLEin the Mexican population (BeltránRamírez et al., 2016).Ciccacci et al. (2014);confirmed the STAT4 rs7574865 variant allele as a risk factor for SLE susceptibility.

\section{Conclusion:-}

The results of the present study in keeping with evidences from literature reveled that although there was no statistically significant association between the STAT4 gene C>G (rs7582694) polymorphism and SLE in the studied group of Egyptian patients, the important role of STAT4 in the pathophysiology of SLE cannot be ruled out. Thus, our results revealed that the same genetic variant has variable role in the same disease among different populations.

\section{References:-}

1. Alarcón-Riquelme, M. E., Ziegler, J. T., Molineros, J., Howard, T. D., et al. (2016): GWAS in an Amerindian ancestry population reveals novel systemic lupus erythematosus risk loci and the role of European admixture.Arthritis \& Rheumatology (Hoboken, NJ),68(4):932-943.

2. BeltránRamírez, O., Mendoza Rincón, J. F., Barbosa Cobos, R. E., Alemán, Á.,Ramírez Bello, J. (2016):STAT4 confers risk for rheumatoid arthritis and systemic lupus erythematosus in Mexican patients. Immunol.Lett.,175:40-43.

3. Bolin, K., Sandling, J. K., Zickert, A., Jönsen, A., et al. (2013): Association of STAT4 polymorphism with severe renal insufficiency in lupus nephritis. PLoS One., 8(12):e84450.

4. Choi, J., Kim, S. T. and Craft, J. (2012): The Pathogenesis of Systemic Lupus Erythematosus - An Update.Current opinion in immunology, 24(6):651-657.

5. Ciccacci, C., Perricone, C., Ceccarelli, F., Rufini, F., et al. (2014): A Multi-locus Genetic Study in a Cohort of Italian SLE Patients Confirms the Association with STAT4 Gene and Describes a New Association with HCP5 Gene. Crispin J, (ed.).PLoS ONE, 9(11):e111991.

6. Cui, Y., Sheng, Y. and Zhang, X. (2013):Genetic susceptibility to SLE: recent progress from GWAS. J Autoimmun., 41:25-33.

7. Delgado-Vega, A., Sánchez, E., Löfgren, S., Castillejo-López, C. andAlarcón-Riquelme, M. E. (2010): Recent Findings on Genetics of Systemic Autoimmune Diseases.Current opinion in immunology, 2(6):698-705.

8. Deng, Y. andTsao, B. P. (2010): Genetic susceptibility to systemic lupus erythematosus in the genomic era.Nat. Rev.Rheumatol., 6(12): 683-692.

9. Gestermann, N.,Mekinian, A., Comets, E., Loiseau, P., et al. (2010):STAT4 is a confirmed genetic risk factor for Sjögren's syndrome and could be involved in type 1 interferon pathway signaling. Genes Immun., 11(5): 432-438.

10. Glas, J., Seiderer, J., Nagy, M., Fries, C., et al. (2010): Evidence forSTAT4as a common autoimmune gene: rs 7574865 is associated with colonic crohn's disease and early disease onset. Unutmaz, D., (ed.).PLoS ONE, 5(4):e10373.

11. Goropevšek, A.,Holcar, M.andAvčin, T. (2016): The Role of STAT Signaling Pathways in the Pathogenesis of Systemic Lupus Erythematosus. Clin Rev Allergy Immunol.,[Epub ahead of print], pubmed: 27216430.

12. Graham, R. R.,Cotsapas, C.,Davies, L.,Hackett, R., et al. (2008): Genetic variants near TNFAIP3 on 6q23 are associated with systemic lupus erythematosus. Nat Genet., 40:1059-1061.

13. Han, J. W., Zheng, H. F.,Cui, Y.,Sun, L. D.,et al. (2009): Genome-wide association study in a Chinese Han population identifies nine new susceptibility loci for systemic lupus erythematosus. Nat. Genet.,41:1234-1237.

14. Harley, J. B.,Alarcón-Riquelme, M. E.,Criswell, L.A.,Jacob, C. O., et al. (2008): Genome-wide association scan in women with systemic lupus erythematosus identifies susceptibility variants inITGAM,PXK, KIAA1542 and other loci.Nat. Genet.,40:204-210. 
15. Hellquist, A., Sandling, J. K., Zucchelli, M., Koskenmies, S., et al. (2010): Variation in STAT4 is associated with systemic lupus erythematosus in a Finnish family cohort. Ann. Rheum. Dis., 69(5):883-6.

16. Hu, W., Wu, S., Zhang, Y., Sigdel, K. R., Lin, Y. andZhong, H. (2016): Association between Toll-Like Receptor 4 Polymorphisms and Systemic Lupus Erythematosus Susceptibility: A Meta-Analysis.BioMed. Research International, 2016:7842587.

17. Li, P.,Cao, C.,Luan, H.,Li, C., et al. (2011):Association of genetic variations in the STAT4 and IRF7/KIAA1542 regions with systemic lupus erythematosus in a Northern Han Chinese population. Hum.Immunol.,72(3):249-255.

18. Luan, H., Li, P., Cao, C., Li, C., et al. (2012):A single-nucleotide polymorphism of the STAT4 gene is associated with systemic lupus erythematosus (SLE) in female Chinese population. Rheumatol. Int.,32(5):12511255 .

19. Nordmark, G., Kristjansdottir, G., Theander, E., Eriksson, P., et al. (2009): Additive effects of the major risk alleles of IRF5 and STAT4 in primary Sjögren's syndrome. Genes Immun.,10(1):68-76.

20. Piotrowski, P.,Lianeri, M., Wudarski, M., Olesińska, M.andJagodziński, P. P. (2012): Contribution of STAT4 gene single-nucleotide polymorphism to systemic lupus erythematosus in the Polish population. Mol. Biol. Rep., 39(9):8861-8866.

21. Sigurdsson, S., Nordmark, G., Garnier, S., Grundberg, E., et al. (2008): A risk haplotype of STAT4 for systemic lupus erythematosus is over-expressed, correlates with anti-dsDNA and shows additive effects with two risk alleles of IRF5. Hum. Mol. Genet.,17(18):2868-2876.

22. Tan, E. M., Cohen, A. D. and Fries, J. F. (1982): The 1982 revised criteria for the classification of systemic lupuserythematosus. Arthritis Rheum., 25:1271-1277.

23. Yang, W.,Shen, N.,Ye, D. Q.,Liu, Q., et al. (2010): Genome-wide association study in Asian populations identifies variants in ETS1 andWDFY4 associated with systemic lupus erythematosus.PLoS Genet.,6:e1000841.

24. Zhou, T. B., Jiang, Z. P., Qin, Y. H.andZhou, J. F. (2014): Retracted Association of STAT4 gene polymorphism with systemic lupus erythematosus / lupus nephritis risk. Nephrology (Carlton),doi: 10.1111/nep.12264. Epub 2014 Apr 16.

25. Zhu, H.,Liu, J., Zhang, H., Wang, Z., et al. (2013):Polymorphisms of STAT4 and the risk of inflammatory bowel disease: A case-control study in Chinese Han population. Biomed. Rep.,1(2): 320-324. 